\title{
Efficacité symbiotique de cinq souches locales de rhizobiums sur les paramètres de croissance du soja
}

\author{
Mako François De Paul N'GBESSO ${ }^{1^{*}}$, Lassina FONDIO ${ }^{1}$, \\ Noupé Diakaria COULIBALY ${ }^{1}$ et $\mathrm{N}^{\prime}$ Guessan Christophe KOUAME ${ }^{2}$ \\ ${ }^{1}$ Centre National de Recherche Agronomique (CNRA), 01 BP 633 Bouaké 01, Côte d'Ivoire. \\ ${ }^{2}$ World Agroforestry Centre (ICRAF) Côte d'Ivoire, Country Program, Cocody Mermoz, 08 BP 2823 Abidjan \\ 08, Côte d'Ivoire. \\ *Auteur correspondant ; E-mail : mako2ngbesso@yahoo.fr; Tel: (+225) 02-02-11-03 / 47-70-00-00
}

\section{REMERCIEMENTS}

Les remerciements les plus sincères des auteurs vont à l'endroit du Projet d'Appui à la Commercialisation et aux Initiatives Locales (PACIL) qui a mis à leur disposition des moyens financiers et techniques pour la réalisation des travaux.

\section{RESUME}

Les travaux de recherche initiés pour remédier à l'absence de nodulation des variétés de soja cultivées en Côte d'Ivoire ont permis d'isoler et de caractériser des souches locales de rhizobium dont cinq ont fait l'objet de cette étude. Le dispositif expérimental était un Split plot avec pour facteur principal les souches de bactéries et pour facteur secondaire les variétés de soja. Les résultats obtenus ont montré qu'au niveau du poids sec de nodules, toutes les variétés à l'exception de Piramama, ont présenté des différences significatives selon la souche de bactérie inoculée. Les poids obtenus ont varié de 4,3 g avec la variété Canarana inoculée par la souche IRAT-FA3 à 10,8 $\mathrm{g}$ avec la variété Boa-Vista inoculée par 26D4. La plupart des variétés n'ont pas répondu à l'inoculation des 3 souches I1C1, 68D2 et 5CB. Le niveau d'insertion des gousses le plus élevé a été de 15,2 cm obtenu avec les plants de la variété Doko inoculée avec la souche 68D2. Comparés au témoin, les délais de floraison de certaines variétés ont présenté des différences significatives selon les traitements. Pour d'autres, les souches inoculées n'ont eu aucun effet. Les délais de maturité étaient compris entre 96,33 jours pour les traitements précoces et 115,3 jours pour les plus tardifs. La plus grande différence de taille à maturité $(18,7 \mathrm{~cm})$ a été observée entre le traitement témoin de Tracaja $(42,4 \mathrm{~cm})$ et son traitement inoculé par la souche 26D4 $(61,1 \mathrm{~cm})$. Les meilleurs niveaux de couverture du sol ont été obtenus avec les traitements issus des souches 26D4 et 81R1.

(C) 2017 International Formulae Group. All rights reserved.

Mots clés : Efficacité symbiotique, souches natives de rhizobiums, soja, Côte d'Ivoire. 


\title{
Symbiotic effectiveness of five local strains of rhizobia on soybean growth parameters
}

\begin{abstract}
The research initiated to remedy the absence of nodulation of soybean cultivated varieties in Côte d'Ivoire has made possible to isolate and characterize local strains of rhizobia. Five of them were the subject of this study. The experimental design was a Split plot. The main factor was bacteria strains and the second factor was soybean varieties. The results obtained upon the weight level of the nodule showed significant differences between all varieties depending on the inoculated strain of bacterium exception of Piramama. The weights obtained varied from $4.3 \mathrm{~g}$ with the Canarana variety inoculated with the strain IRAT-FA3 to $10.8 \mathrm{~g}$ with the Boa-Vista variety inoculated with 26D4. Most varieties did not respond to inoculation of the 3 strains I1C1, $68 \mathrm{D} 2$ and $5 \mathrm{CB}$. The highest pod insertion level was $15.2 \mathrm{~cm}$ obtained with the plants of Doko variety inoculated with 68D2 strains. Compared to the control, the flowering period of certain varieties showed significant differences according to the treatments. For others, the inoculated strains had no effect. The maturity periods are between 96.33 days for early treatments and 115.3 days for the later ones. The largest difference in plant size of $(18.7 \mathrm{~cm})$ was observed between the control treatment of Tracaja $(42.4 \mathrm{~cm})$ and its treatment inoculated with strain 26D4 $(61.1 \mathrm{~cm})$. The best soil cover levels were obtained with treatments from strains 26D4 and 81R1.
\end{abstract}

(C) 2017 International Formulae Group. All rights reserved.

Keywords: Symbiotic effectiveness, Native rhizobia strains, Soybeans, Côte d'Ivoire.

\section{INTRODUCTION}

Une étude réalisée sur la culture du soja a révélé que la plupart des variétés cultivées en Côte d'Ivoire ne produisent pas de nodules ou en produisent très peu (Kouamé et al., 2002). Or, selon (Anikwe et al., 2003), pour qu'il ait la formation de nodules, il faut que la souche bactérienne soit présente en quantité suffisante dans le sol et surtout, qu'elle soit compatible avec la variété en présence. Aussi, pourrait-on émettre l'hypothèse selon laquelle l'absence ou le nombre réduit des populations de bactéries locales serait à l'origine responsable de l'absence observée de nodules. Ce qui pourrait ainsi, expliquer le faible développement végétatif des plants et des rendements de moins d'une tonne à hectare obtenus au niveau de cette culture chez les producteurs. Des tests d'inoculation par enrobage des semences avant le semis avec une souche introduite de Bradyrhizobium japonicum (IRAT-FA3) ont permis de lever effectivement cette équivoque par l'accroissement du nombre de nodules au niveau des variétés cultivées (N'Gbesso et al., 2010). De plus, des travaux ultérieurs ont montré que les plants issus des graines inoculées présentent un bon développement végétatif et des rendements élevés. En effet, les écarts de rendement obtenus entre les traitements inoculés et non inoculés en fonction des localités, ont varié entre 9,29\% et $52,31 \%$ selon le site (N'Gbesso, 2011). Dès lors, l'inoculation des semences de soja par des souches efficientes de bactéries avant le semis a été recommandée dans l'itinéraire technique de la culture du soja en Côte d'Ivoire. Il s'est donc avéré nécessaire d'identifier des souches natives de bactéries plus efficientes et qui permettront d'améliorer les rendements et le développement végétatif des plants en lieu et place de la souche introduite. C'est dans cette optique que plusieurs souches locales de bactéries du genre Bradyrhizobium ont été isolées et caractérisées. Cinq d'entre elles ont fait l'objet de cette étude dont l'objectif est d'identifier parmi les souches natives isolées, celles qui améliorent significativement les paramètres de croissance des variétés de soja cultivées en Côte d'Ivoire comparativement à la souche introduite IRAT-FA3.

\section{MATERIEL ET METHODES Milieu d'étude}


Les travaux ont été conduits à la Station de Recherche sur les Cultures Vivrières du Centre National de Recherche Agronomique (SRCV/CNRA) à Bouaké entre juillet et décembre 2013. Cette période a été définie par Kouamé et al. (1994) comme étant la plus propice à la culture du soja en Côte d'Ivoire. Les sols de cette station expérimentale sont ferralitiques typiques ou rajeunis, sur granite, ou des sols remaniés, avec des sous-groupes indurés, appauvris ou hydromorphes (Koné et al., 2009). La composition chimique des sols telle décrite par Fondio et al. (2013) est présentée dans le Tableau 1.

Bouaké, ville du Centre de la Côte d'Ivoire à environ $350 \mathrm{~km}$ d'Abidjan, est située à $7^{\circ} 46$ de Latitude Nord, $5^{\circ} 06$ de Longitude Ouest et à $376 \mathrm{~m}$ d'altitude (Figure 1). Elle a une superficie de $28.530 \mathrm{~km}^{2}$ avec une densité de 53,9 habitants $/ \mathrm{km}^{2}$ (World, 2013). Sa population est majoritairement paysanne (Ouattara, 2001).

Le climat est de type guinéen forestier avec un régime pluviométrique bimodal. La pluviométrie moyenne annuelle est comprise entre 724 et $1423 \mathrm{~mm}$. Mais chacune de ces périodes est de moins en moins marquée ces dernières années (Brou et al., 2005 ; Soro et al., 2007). Le relief se présente essentiellement sous forme de plateaux avec des altitudes variant entre 200 à $500 \mathrm{~m}$ (Avit et al., 1999).

\section{Matériel végétal}

Six (06) variétés de soja sélectionnées parmi plusieurs introduites du Brésil et du Nigeria depuis un certain nombre d'années pour certaines et récemment pour d'autres ont été utilisées pour conduire cet essai (Tableau 2).

Au niveau des 6 souches de bactéries étudiées, on note :

- cinq (05) sont d'origines locales. Il s'agit des souches I1C1; 5CB ; 81R1; 26D4 et $68 \mathrm{D} 2$ (Tableau 3 ). Elles ont été isolées à partir d'échantillons de sols prélevés dans différentes localités de Côte d'Ivoire. Puis, elles ont été caractérisées au Laboratoire des Symbioses Tropicales à Montpellier (LSTM).
- une (01) souche IRAT-FA3 a servi de référence. Elle avait été introduite du Laboratoire des Symbioses Tropicales de Montpellier depuis 2000. Son efficience sur les variétés de soja cultivées en Côte d'Ivoire a été établie par N'Gbesso et al. (2010).

En plus de ces souches, un traitement neutre ou témoin ( $\mathrm{T}$ : sans inoculum) a servi à tester la présence ou non de souches natives de bactéries dans la parcelle expérimentale (Tableau 3).

\section{Méthodes}

Les souches de bactéries utilisées sont des bactéries du genre Bradyrhizobium spécialement sélectionnées pour stimuler au maximum la fixation biologique de l'azote en culture de soja. Elles ont été conditionnées sous la forme concentrée dans des sachets de $100 \mathrm{~g}$ de tourbe stérilisée appelée inoculum. Pour avoir leur efficacité, chaque souche de bactéries a été inoculée par enrobage des graines des différentes variétés de soja avant le semis (Tableau 3).

Le dispositif expérimental était un Split plot à deux facteurs avec 3 répétitions. Le facteur principal était représenté par les souches de bactéries ou inoculum. Ce facteur, avec sept (7) niveaux de traitements dont 5 sont constitués des 5 souches locales de bactéries étudiées, de la souche de référence IRAT-FA3 et d'un témoin neutre (T) sans inoculum. Quant au facteur secondaire, il était représenté par les six (6) variétés de soja que sont: Canarana, Doko, Piramama, Tracaja, IT-235 et Boa-Vista. La combinaison entre les niveaux de traitements de ces deux facteurs ( 7 $x$ 6) donne un total de 42 parcelles élémentaires par répétition. En tenant compte des 3 répétitions, cela donne 126 parcelles élémentaires pour l'ensemble de l'essai.

Dans chaque bloc, la parcelle élémentaire était représentée par 9 lignes de semis sur une superficie de $4 \mathrm{~m} \times 4 \mathrm{~m}$, soit 16 $\mathrm{m}^{2}$. Les deux lignes de semis situées de chaque côté de la parcelle élémentaire ont constitué la bordure pour celle-ci. Ainsi, la parcelle utile était limitée aux $9 \mathrm{~m}^{2}$ intérieurs. Deux traitements consécutifs étaient distants de $1 \mathrm{~m}$. Tandis que deux blocs consécutifs ont été séparés par une allée de $2 \mathrm{~m}$ de large. La 
superficie totale de l'essai avec les bordures était de $2500 \mathrm{~m}^{2}$.

Les observations et mesures ont été effectuées selon le manuel d'évaluation variétale de soja de l'Asian Vegetable Research Center (AVRDC, 2009). Elles ont porté sur les paramètres symbiotiques de croissance suivants :

- l'incidence de l'inoculation des souches sur les dates phénologiques (délais de floraison et de maturité) des variétés de soja; - la capacité de nodulation des souches au niveau des racines. Elle a été évaluée à travers les poids sec de nodules prélevés sur 20 plants 60 jours après semis (JAS).

- la hauteur d'insertion des premières gousses sur la tige principale
- la taille des plants à maturité ;

- l'envergure des plants au stade du début de formation des gousses.

Analyse statistique

L'analyse de certaines données a été faite à l'aide du logiciel SAS selon une ANOVA II, suivie de la séparation des moyennes à l'aide du test de Duncan au seuil de $5 \%$. En ce qui concerne le poids de nodules prélevés sur 20 plants, des histogrammes ont été faits avec le logiciel Excel. A ce niveau, les moyennes ont été séparées à l'aide du Test LSD de Fisher au seuil de 5\%.
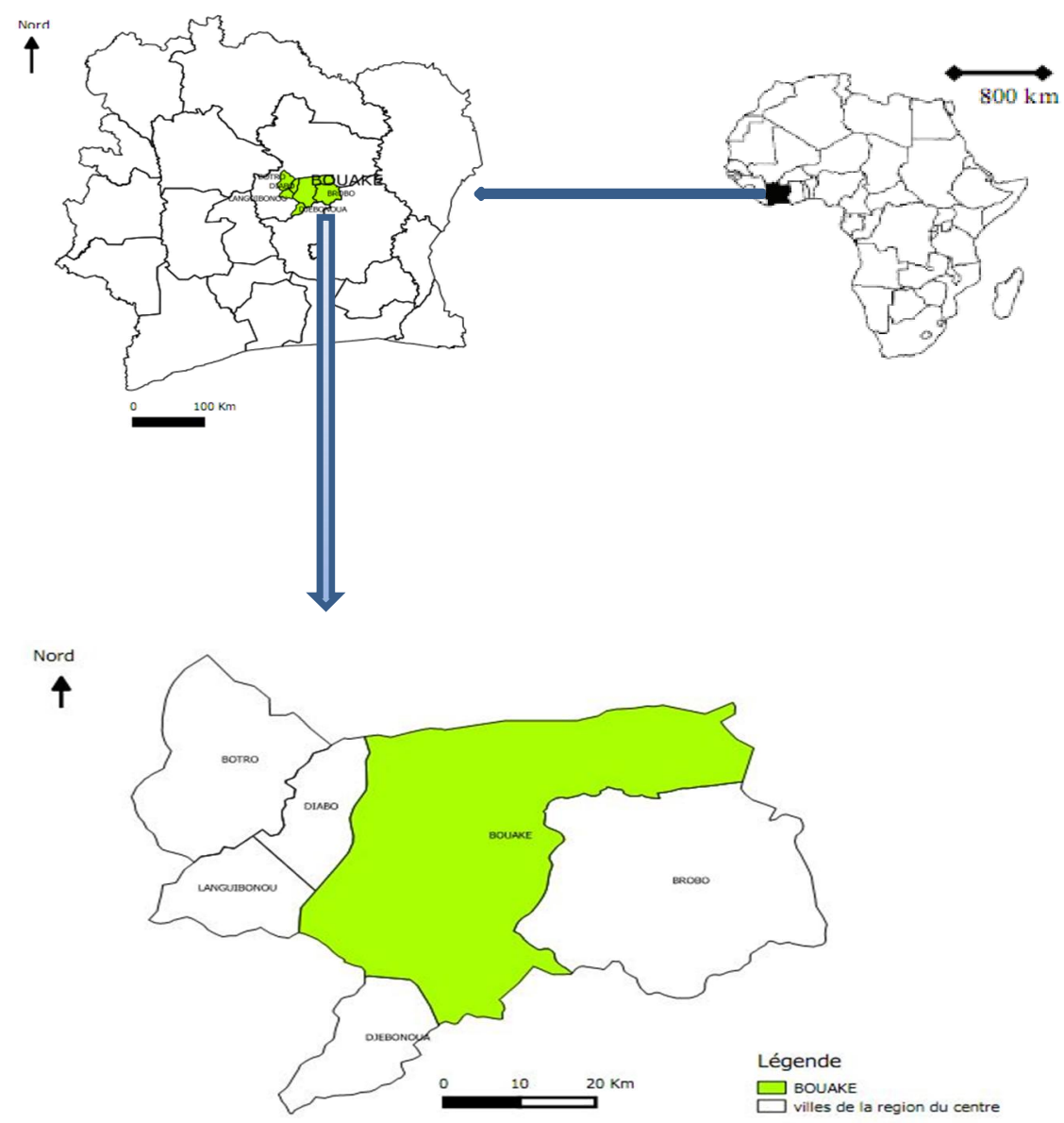

Figure 1 : Localisation de la zone d'étude (Source : Brindou, 2016). 
Tableau 1 : Composition chimique du sol de la station d'expérimentation.

\begin{tabular}{|c|c|c|c|c|c|c|c|c|}
\hline \multirow[t]{2}{*}{$\mathbf{P h}$} & \multirow[t]{2}{*}{$\mathbf{C} / \mathbf{N}$} & \multicolumn{3}{|c|}{ Phosphore (ppm) } & \multicolumn{4}{|c|}{ Complexe absorbant (cmol/kg) } \\
\hline & & P total & P. ass. & CEC & $\mathrm{Ca}^{2+}$ & $\mathrm{Mg}^{2+}$ & $\mathrm{K}^{+}$ & $\mathrm{Na}^{+}$ \\
\hline 4,5 & 10,5 & 313 & 100 & 4,72 & 0,322 & 0,129 & 0,072 & 0,039 \\
\hline
\end{tabular}

Tableau 2 : Liste des variétés de soja étudiées.

\begin{tabular}{cll}
\hline Variété & Origine & \multicolumn{1}{c}{ Observation } \\
\hline Canarana & Brésil & $\begin{array}{l}\text { Variétés considérées comme étant des variétés locales car } \\
\text { introduites et cultivées depuis les années } 1990 \text { en Côte } \\
\text { d'Ivoire }\end{array}$ \\
Poko & Variétés introduites en 2007, évaluées et en phase de \\
Tracaja & Brésil & prévulgarisation, \\
IT-235 & Variété introduite de l'International Institute for Tropical \\
& Agriculture (IITA) basé au Nigeria. Cette lignée est en \\
& phase de sélection finale, \\
Boa-Vista & Variété introduite en 2007, évaluée et en phase de \\
& prévulgarisation
\end{tabular}

Tableau 3: Liste des souches de bactéries étudiées.

\begin{tabular}{lll}
\hline Souche & Spécification & Observation \\
\hline I1C1 & Bradyrhizobium japonicum USDA115 & \\
$5 \mathrm{CB}$ & Bradyrhizobium elkanii $\mathrm{R} 17$ & \\
$81 \mathrm{R} 1$ & Bradyrhizobium elkanii UNDA90 & Natives de Côte \\
$26 \mathrm{D} 4$ & d'Ivoire \\
$68 \mathrm{D} 2$ & Bradyrhizobium japonicum DASA38025 & \\
IRAT-FA3 & Bradyrhizobium japonicum & Introduite (France) \\
\hline
\end{tabular}




\section{RESULTATS}

Incidence des souches sur les délais de floraison et de maturité des variétés de soja

De façon générale, les délais de floraison pour tous les traitements se sont situés entre 48 et 51 jours (Tableau 4). Comparés au témoin, les délais de floraison des variétés de soja testées ont présenté pour certaines, quelques différences significatives selon les traitements qui leur ont été appliquées. Tandis que pour d'autres, les différentes souches de bactéries inoculées n'ont eu aucun effet sur leur délai de floraison initial. Ainsi, au niveau de la variété Canarana, l'inoculation avec la souche de rhizobium 81R1 a rendu précoce la floraison avec 48 jours quand les autres traitements ont affiché entre 50 et 51 jours. Chez la variété Doko, c'est le traitement témoin qui, au contraire, a retardé la floraison de 2 jours par rapport aux 6 souches de bactéries. Le temps de floraison normal de 50 jours affiché par le traitement témoin a donc été réduit à 48 jours chez cette variété. Par contre chez la variété Piramana, c'est la souche 68D2 qui a retardé de façon significative la floraison de 3 jours. Ce délai est passé ainsi de 48 jours à 51 jours. Contrairement à ces 3 variétés, les 3 autres que sont Tracaja, IT-235 et Boa-Vista n'ont présenté aucune différence significative au niveau de leur délai de floraison quel que soit le traitement. Ainsi, ces délais ont été de 48 jours pour la variété Tracaja et 51 jours pour les variétés IT-235 et Boa-Vista. Au niveau des moyennes, on note qu'il n'y a pas de différences significatives entre tous les traitements y compris le témoin (Tableau 4).

Les délais de maturité des différents traitements se situent entre 96,33 jours pour les plus précoces et 115,3 jours pour les plus tardifs (Tableau 5). A ce niveau, on s'aperçoit que la variété Canarana inoculée avec la souche 81R1a eu son cycle réduit de deux jours par rapport au traitement témoin. De même, au niveau de la variété Doko, ce sont les traitements avec les souches 26D4 et 81R1 qui ont induit une réduction d'environ 2 jours sur le cycle. Elle est Passée ainsi de 106 jours affichés par le traitement témoin à 104 jours. La variété Piramana n'a subi aucune influence des traitements effectués par les différentes souches. Le délai de maturité d'environ 97 jours n'a pas varié de façon significative par rapport au témoin. Il en a été de même de la variété IT-235. En effet, celle-ci a vu son délai de maturité varier entre 105 et 106 jours. Au niveau de la variété Tracaja, c'est la souche $81 \mathrm{R} 1$ qui a réduit le delai de maturité de près de 3 jours par rapport aux autres traitements. De même, chez la variété Boa-Vista la souche $81 R 1$ a affiché 112 jours au lieu de 115 pour le témoin.

En moyenne, les délais de maturité ont varié entre 105,44 jours avec la souche 81R1 et 107 jours chez le témoin. Comme pour le délai de floraison, on s'est aperçu globalement que l'incidence des souches de bactéries sur les délais de maturité n'a pas été très marquée.

Incidence des souches sur la production de nodules par les variétés de soja

En ce qui concerne le poids sec de nodules prélevés sur 20 plants, les histogrammes peuvent être classés en deux groupes. Le premier groupe, généré par les souches $\mathrm{I} 1 \mathrm{C} 1,68 \mathrm{D} 2$ et $5 \mathrm{CB}$ ont été très proches de ceux du témoin. Ces histogrammes sont de faibles amplitudes. Le deuxième groupe, constitué d'histogrammes à fortes amplitudes, est issu des souches 26D4, IRATFA3 et 81R1 (Figure 2).

$\mathrm{Au}$ sein des variétés, des différences significatives ont été observées selon le traitement appliqué sauf la variété Piramana qui est restée constante face à tous les traitements y compris le traitement témoin. Ainsi, au niveau des variétés Canarana, Doko, Tracaja, IT-235 et Boa-Vista, ce sont les souches 26D4, IRAT-FA3 et 81R1 qui ont induit les poids de nodules secs les plus élevés. Ce qui traduit leurs capacités élevée de production de nodules. Les poids secs 
obtenus vont de $4,3 \mathrm{~g}$ avec la variété Canarana inoculée par la souche IRAT-FA3 à 10,8 g chez la variété Boa-Vista inoculée avec la souche 26D4.

On a remarqué au niveau de la même Figure 2 que ces mêmes variétés ont produit peu de nodules avec les souches I1C1, 68D2 et $5 \mathrm{CB}$. Les amplitudes des histogrammes sont proches de ceux du traitement témoin (sans inoculum). De plus, on a observé que dans l'ensemble, les variétés évaluées ont été très peu sensibles ou pas du tout à l'inoculation par ces trois souches.

\section{Incidence des souches sur la hauteur d'insertion des premières gousses sur la tige principale}

Vis-à-vis des différentes variétés, la plupart des souches d'inoculum ont présenté des différences significatives au niveau de la hauteur d'insertion des premières gousses sur la tige principale exceptés le traitement témoin et la souche $81 \mathrm{R} 1$. Le niveau d'insertion des gousses le plus élevé a été de $15,2 \mathrm{~cm}$ obtenu avec les plants de la variété Doko inoculée avec la souche 68D2. Les variétés Piramana, Canarana et IT-235 inoculées par la souche I1C1 d'une part et d'autre part la variété Canarana inoculée avec la souche 68D2 ont eu les gousses les plus basses car elles sont situées seulement à 6,27 $\mathrm{cm}$ et $7,4 \mathrm{~cm}$ du sol (Tableau 6).

Selon la norme, les meilleurs niveaux d'insertion sont ceux qui sont situés au-delà de $8 \mathrm{~cm} \mathrm{du}$ sol. Ainsi, la plupart des valeurs étant largement au-dessus de $10 \mathrm{~cm}$, le comportement des variétés vis-à-vis des souches peut être considéré comme étant satisfaisant. En effet, la nécessité de sélectionner des variétés dont les premières gousses sont situées au-dessus de $8 \mathrm{~cm}$ du sol est donc très importante si l'on veut éviter des pertes de récolte en culture mécanisée.
Incidence des souches sur la longueur de la tige des variétés de soja à maturité

Au niveau de ce paramètre, nous avons noté que les souches de bactéries 26D4 et 81R1 améliorent de façon significative la croissance de la plupart des variétés étudiées par rapport au traitement témoin. Par contre, les souches $11 \mathrm{C} 1,68 \mathrm{D} 2$ et $5 \mathrm{CB}$ n'ont induit des différences de croissance des tiges qu'au niveau des variétés Tracaja et IT-235. Les autres variétés comme Doko, Piramana et Boa-Vista n'ont pas vu leur taille améliorée significativement par ces souches si on se réfère aux valeurs affichées par le traitement témoin (Tableau 7). On a pu remarquer ainsi, que la plus grande différence de taille à maturité $(18,7 \mathrm{~cm})$ a été observée entre le traitement témoin de Tracaja $(42,4 \mathrm{~cm})$ et son traitement inoculé par la souche 26D4 $(61,1 \mathrm{~cm})$.

Les plus fortes moyennes de croissance de tige ont été obtenues avec les souches $26 \mathrm{D} 4(55,90 \mathrm{~cm})$ et $81 \mathrm{R} 1(53 \mathrm{~cm})$. La plus faible moyenne a été obtenue avec le traitement témoin $(47,8 \mathrm{~cm})$.

\section{Incidence des souches sur la capacité de} couverture du sol des variétés de soja

Les meilleurs niveaux de couverture du sol, au stade du début de la formation des gousses ont été obtenus au niveau des six variétés avec les traitements issus des souches $26 \mathrm{D} 4$ et $81 \mathrm{R} 1$. Au niveau de ces variétés, les taux de couverture du sol ont varié entre 3,5/5 et $4,8 / 5$. Quant aux autres souches, les réactions divergent en fonction de la variété en présence (Tableau 8). En général, les plants $\mathrm{du}$ traitement témoin ont présenté une mauvaise couverture du sol sauf ceux de la variété Piramana qui ont obtenu une note de 4,3/5. De plus, cette variété a présenté la meilleure moyenne au niveau de ce paramètre avec une note de 3,8/5. Elle a été suivie des variétés Tracaja et IT-235 avec des notes de $3,3 / 5$ et 3,5/5, respectivement (Tableau 8). 
Tableau 4: Délais de floraison (jour) des différentes variétés de soja.

\begin{tabular}{lccccccc}
\hline & 26D4 & $\begin{array}{l}\text { IRAT- } \\
\text { FA3 }\end{array}$ & I1C1 & 68D2 & Témoin & 5CB & $\mathbf{8 1 R 1}$ \\
\hline Canarana & $50^{\mathrm{a}}$ & $51^{\mathrm{a}}$ & $50^{\mathrm{a}}$ & $50^{\mathrm{a}}$ & $51^{\mathrm{a}}$ & $51^{\mathrm{a}}$ & $48^{\mathrm{b}}$ \\
Doko & $48^{\mathrm{b}}$ & $48^{\mathrm{b}}$ & $48^{\mathrm{b}}$ & $48^{\mathrm{b}}$ & $50^{\mathrm{a}}$ & $48^{\mathrm{b}}$ & $48^{\mathrm{b}}$ \\
Piramana & $48^{\mathrm{b}}$ & $48^{\mathrm{b}}$ & $48^{\mathrm{b}}$ & $51^{\mathrm{a}}$ & $48^{\mathrm{b}}$ & $48^{\mathrm{b}}$ & $48^{\mathrm{b}}$ \\
Tracaja & $48^{\mathrm{b}}$ & $48^{\mathrm{b}}$ & $48^{\mathrm{b}}$ & $48^{\mathrm{b}}$ & $48^{\mathrm{b}}$ & $48^{\mathrm{b}}$ & $48^{\mathrm{b}}$ \\
IT-235 & $51^{\mathrm{a}}$ & $51^{\mathrm{a}}$ & $51^{\mathrm{a}}$ & $51^{\mathrm{a}}$ & $51^{\mathrm{a}}$ & $51^{\mathrm{a}}$ & $51^{\mathrm{a}}$ \\
Boa-Vista & $51^{\mathrm{a}}$ & $51^{\mathrm{a}}$ & $51^{\mathrm{a}}$ & $51^{\mathrm{a}}$ & $51^{\mathrm{a}}$ & $51^{\mathrm{a}}$ & $51^{\mathrm{a}}$ \\
\hline Moyenne & 49,33 & 49,50 & 49,33 & 49,67 & 49,83 & 49,5 & 49,0 \\
CV & 1,43 & 0 & 1,43 & 1,80 & 1,42 & 0 & 0 \\
$\mathrm{R}^{2}$ & 0,87 & 1,0 & 0,87 & 0,80 & 0,87 & 1 & 1 \\
\hline
\end{tabular}

*Les moyennes avec la même lettre ne sont pas très différentes

Tableau 5 : Délais de maturité (jour) des différentes variétés de soja.

\begin{tabular}{|c|c|c|c|c|c|c|c|}
\hline & 26D4 & IRAT-FA3 & I1C1 & 68D2 & Témoin & $5 \mathrm{CB}$ & 81R1 \\
\hline Canarana & $110^{\mathrm{a}}$ & $110^{\mathrm{a}}$ & $110^{\mathrm{b}}$ & $110,0^{b}$ & $110,0^{\mathrm{b}}$ & $110^{\mathrm{ab}}$ & $108,33^{b}$ \\
\hline Doko & $104,33^{b}$ & $106^{\mathrm{b}}$ & $106,67^{\mathrm{b}}$ & $106,67^{\mathrm{b}}$ & $106,0^{b c}$ & $106,66^{\mathrm{b}}$ & $104,33^{\mathrm{c}}$ \\
\hline Piramana & $97,33^{\mathrm{c}}$ & $97,67^{\mathrm{c}}$ & $97,67^{\mathrm{c}}$ & $99,33^{\mathrm{c}}$ & $97,67^{\mathrm{d}}$ & $96,66^{\mathrm{c}}$ & $96,33^{\mathrm{d}}$ \\
\hline Tracaja & $110^{\mathrm{a}}$ & $110^{\mathrm{a}}$ & $108,33^{b}$ & $110,0^{\mathrm{b}}$ & $108,33^{b c}$ & $106,66^{\mathrm{b}}$ & $106,67^{\mathrm{bc}}$ \\
\hline IT-235 & $105^{\mathrm{b}}$ & $105^{b}$ & $106,67^{b}$ & $106,67^{b}$ & $105,0^{\mathrm{c}}$ & $106,66^{\mathrm{b}}$ & $105^{\mathrm{bc}}$ \\
\hline Boa-Vista & $111^{\mathrm{a}}$ & $112^{\mathrm{a}}$ & $114^{\mathrm{a}}$ & $115,3^{\mathrm{a}}$ & $115,0^{\mathrm{a}}$ & $114^{\mathrm{a}}$ & $112^{\mathrm{a}}$ \\
\hline Moyenne & 106,28 & 106,78 & 107,22 & 108 & 107,0 & 106,78 & 105,44 \\
\hline $\mathrm{CV}$ & 0,90 & 1,56 & 1,87 & 1,94 & 2,05 & 2,27 & 1,67 \\
\hline $\mathrm{R}^{2}$ & 0,98 & 0,94 & 0,92 & 0,91 & 0,91 & 0,89 & 0,93 \\
\hline
\end{tabular}




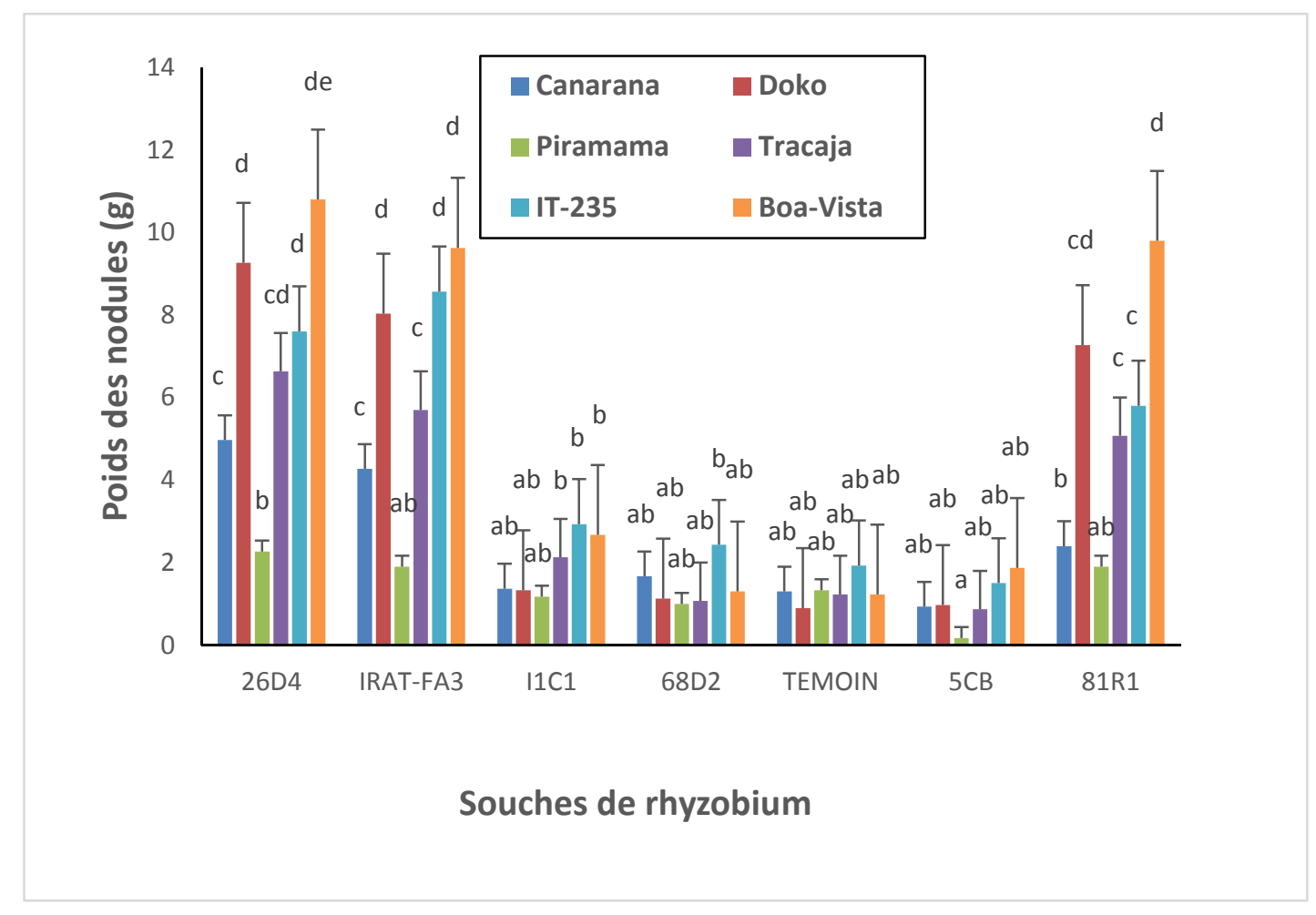

Figure 2 : Poids de nodules secs prélevés sur 20 plants.

Tableau 6 : Niveau d'insertion $(\mathrm{cm})$ des premières gousses sur la tige principale.

\begin{tabular}{llllllll}
\hline & 26D4 & IRAT-FA3 & I1C1 & $\mathbf{6 8 D 2}$ & TEMOIN & 5CB & $\mathbf{8 1 R 1}$ \\
\hline Canarana & $10,27^{\mathrm{b}}$ & $9,33^{\mathrm{b}}$ & $6,27^{\mathrm{c}}$ & $7,4^{\mathrm{b}}$ & $11,33^{\mathrm{a}}$ & $8,67^{\mathrm{c}}$ & $8,87^{\mathrm{a}}$ \\
Doko & $12,33^{\mathrm{a}}$ & $12,93^{\mathrm{a}}$ & $12,4^{\mathrm{a}}$ & $15,2^{\mathrm{a}}$ & $11,13^{\mathrm{a}}$ & $12,47^{\mathrm{a}}$ & $12,53^{\mathrm{a}}$ \\
Piramana & $8,87^{\mathrm{b}}$ & $11,07^{\mathrm{ab}}$ & $7,87^{\mathrm{bc}}$ & $8,27^{\mathrm{b}}$ & $8,80^{\mathrm{a}}$ & $9,33^{\mathrm{bc}}$ & $9,67^{\mathrm{a}}$ \\
Tracaja & $9,2^{\mathrm{b}}$ & $11,47^{\text {ab }}$ & $13,33^{\mathrm{a}}$ & $14,4^{\mathrm{a}}$ & $11,27^{\mathrm{a}}$ & $11,53^{\mathrm{ab}}$ & $9,0^{\mathrm{a}}$ \\
IT-235 & $8,67^{\mathrm{b}}$ & $10,80^{\mathrm{ab}}$ & $7,4 \mathrm{~b}^{\mathrm{c}}$ & $9,33^{\mathrm{b}}$ & $10,0^{\mathrm{a}}$ & $8,07^{\mathrm{c}}$ & $9,47^{\mathrm{a}}$ \\
Boa-Vista & $12,67^{\mathrm{a}}$ & $10,08^{\mathrm{ab}}$ & $9,53^{\mathrm{b}}$ & $11,33^{\mathrm{ab}}$ & $10,73^{\mathrm{a}}$ & $13,8^{\mathrm{a}}$ & $10,80^{\mathrm{a}}$ \\
\hline Moyenne & 9,67 & 10,78 & 9,8 & 11,08 & 10,54 & 10,64 & 10,5 \\
CV & 11,98 & 18,26 & 12,97 & 18,95 & 24,13 & 12,51 & 21,14 \\
$\mathrm{R}^{2}$ & 0,87 & 0,51 & 0,91 & 0,83 & 0,38 & 0,82 & 0,49 \\
\hline
\end{tabular}


Tableau 7 : Longueur de la tige $(\mathrm{cm})$ à maturité des différentes variétés.

\begin{tabular}{llllllll}
\hline & $26 \mathrm{D} 4$ & IRAT-FA3 & I1C1 & $68 \mathrm{D} 2$ & Témoin & $5 \mathrm{CB}$ & $81 \mathrm{R} 1$ \\
\hline Canarana & $42,467 \mathrm{~b}$ & $46,87^{\mathrm{a}}$ & $46,27^{\mathrm{a}}$ & $42,93^{\mathrm{b}}$ & $50,53^{\mathrm{ab}}$ & $41,80^{\mathrm{b}}$ & $51,87^{\mathrm{ab}}$ \\
Doko & $54,4 \mathrm{a}$ & $49,33^{\mathrm{a}}$ & $47,47^{\mathrm{a}}$ & $51,47^{\mathrm{ab}}$ & $47,0^{\mathrm{ab}}$ & $45,8^{\mathrm{ab}}$ & $47,87^{\mathrm{b}}$ \\
Piramana & $58,6 \mathrm{a}$ & $52,60^{\mathrm{a}}$ & $56,60^{\mathrm{a}}$ & $44,13^{\mathrm{ab}}$ & $54,67^{\mathrm{a}}$ & $50,7^{\mathrm{ab}}$ & $49,0^{\mathrm{b}}$ \\
Tracaja & $61,07^{\mathrm{a}}$ & $47,47^{\mathrm{a}}$ & $45,93^{\mathrm{a}}$ & $50,40^{\mathrm{ab}}$ & $42,40^{\mathrm{b}}$ & $53,2^{\mathrm{ab}}$ & $55,73^{\mathrm{ab}}$ \\
IT-235 & $57,0^{\mathrm{a}}$ & $56,53^{\mathrm{a}}$ & $52,73^{\mathrm{a}}$ & $56,73^{\mathrm{a}}$ & $47,93^{\mathrm{ab}}$ & $52,0^{\mathrm{ab}}$ & $60,53^{\mathrm{a}}$ \\
Boa-Vista & $61,87^{\mathrm{a}}$ & $48,0^{\mathrm{a}}$ & $45,33^{\mathrm{a}}$ & $55,47^{\mathrm{ab}}$ & $44,27^{\mathrm{ab}}$ & $55,0^{\mathrm{a}}$ & $53,0^{\mathrm{ab}}$ \\
\hline Moyenne & 55,90 & 50,13 & 49,05 & 50,19 & 47,80 & 49,79 & 53,0 \\
CV & 10,51 & 15,37 & 12,74 & 13,45 & 11,38 & 13,10 & 10,76 \\
$\mathrm{R}^{2}$ & 0,72 & 0,40 & 0,45 & 0,58 & 0,63 & 0,48 & 0,51 \\
\hline
\end{tabular}

*Les moyennes avec la même lettre ne sont pas très différentes

Tableau 8 : Niveau de couverture du sol des plants au stade du début de formation des gousses (1 à $5)$.

\begin{tabular}{llllllll}
\hline & 26D4 & IRAT-FA3 & I1C1 & $\mathbf{6 8 D 2}$ & Témoin & $\mathbf{5 C B}$ & $\mathbf{8 1 R 1}$ \\
\hline Canarana & $3,0^{\mathrm{a}}$ & $3,0^{\mathrm{ab}}$ & $2,67^{\mathrm{ab}}$ & $3,0^{\mathrm{a}}$ & $1,67^{\mathrm{b}}$ & $1,33^{\mathrm{b}}$ & $4,0^{\mathrm{a}}$ \\
Doko & $3,0^{\mathrm{a}}$ & $2,67^{\mathrm{ab}}$ & $2,0^{\mathrm{b}}$ & $2,67^{\mathrm{a}}$ & $1,67^{\mathrm{b}}$ & $3,0^{\mathrm{ab}}$ & $3,67^{\mathrm{a}}$ \\
Piramana & $4,33^{\mathrm{a}}$ & $3,33^{\mathrm{ab}}$ & $3,33^{\mathrm{a}}$ & $2,0^{\mathrm{a}}$ & $4,0^{\mathrm{a}}$ & $3,67^{\mathrm{a}}$ & $3,67^{\mathrm{a}}$ \\
Tracaja & $4,67^{\mathrm{a}}$ & $2,67^{\mathrm{ab}}$ & $2,33^{\mathrm{b}}$ & $2,33^{\mathrm{a}}$ & $2,0^{\mathrm{b}}$ & $2,67^{\mathrm{ab}}$ & $3,67 \mathrm{a}$ \\
IT-235 & $3,0^{\mathrm{a}}$ & $3,67^{\mathrm{a}}$ & $2,67^{\mathrm{ab}}$ & $3,0^{\mathrm{a}}$ & $2,33^{\mathrm{b}}$ & $3,0^{\mathrm{ab}}$ & $3,67^{\mathrm{a}}$ \\
Boa-Vista & $4,0^{\mathrm{a}}$ & $2,0^{\mathrm{b}}$ & $2,0^{\mathrm{b}}$ & $3,0^{\mathrm{a}}$ & $2,0^{\mathrm{b}}$ & $3,33^{\mathrm{a}}$ & $3,0^{\mathrm{a}}$ \\
\hline Moyenne & 3,67 & 2,89 & 2,50 & 2,67 & 2,28 & 2,83 & 3,61 \\
CV & 22,81 & 23,36 & 19,32 & 26,51 & 24,92 & 32,22 & 27,54 \\
$\mathrm{R}^{2}$ & 0,56 & 0,67 & 0,64 & 0,5 & 0,79 & 0,55 & 0,19 \\
\hline \multicolumn{2}{r}{ *Les moyennes avec la même lettre ne sont pas très différentes. }
\end{tabular}

\section{DSCUSSION}

Les variations observées dans les délais de floraison et de maturité au niveau de certaines variétés vis-à-vis de certains traitements trouvent une explication dans l'efficience des bactéries avec lesquelles elles ont été inoculées. En effet selon (N'Gbesso ; 2010), en face d'une souche efficiente de bactéries, les paramètres morphologiques et physiologiques d'une variété compatible se trouvent améliorés au point d'induire une précocité dans les délais de floraison et de maturation. De même Woomer (2010), dans des expérimentations similaires a montré que des souches autochtones de bactérie isolées réduisent de façon significative les paramètres 
phénologiques que sont les délais de floraison et de maturation. Selon le même auteur, une inoculation efficiente induit une amélioration significative de la coloration des feuilles passant de vert clair à vert foncé et des plants de grande taille. Ceci serait dû à la fixation symbiotique de l'azote assurée par une importante population de bactéries enfouie dans le sol avec l'inoculation. En effet, selon Lecourtier (2016), une inoculation typique des graines de soja induit une augmentation de $256 \times 10^{9}$ bactéries par are dans le sol. Cette bonne nutrition azotée pourrait être responsable des modifications observées au niveau des déliais de floraison et de maturité. Il en est de même chez le niébé où Gbaguidi et al. (2015) ont montré qu'il existe des corrélations positives existent entre ces deux paramètres.

La production de nodules est un facteur essentiel pour la réalisation d'une relation symbiotique efficiente. Leur absence ou insuffisance annule ou réduit le processus de fixation biologique de l'azote (Aboubacar et al., 2013). La présence de nodules est souvent le signe d'une compatibilité entre une variété de soja et la souche de bactérie en présence. Ainsi, les faibles poids secs de nodules obtenus avec les souches I1C1, 68D2 et 5CB et surtout avec le traitement témoin pourraient être le fait d'une nodulation spontanée avec des souches natives de Bradyrhizobium japonicum et non des souches inoculées. Cette présence de nodules chez les témoins est synonyme de l'existence de souches autochtones de Bradyrhizobium japonicum dans le sol du site d'étude. Les quantités peu élevée voire nulles de nodules spontanés obtenues chez certaines variétés seraient liées à la faible densité de population de ces souches dans le sol de ce site (N'Gbesso et al., 2013). De même, Mulongoy (2005), parlant des facteurs biotiques limitants de la nodulation, a cité soit l'absence de souches efficientes requises soit leur insuffisance comme une des contraintes majeures de ce phénomène biologique. Ces comportements des variétés vis-à-vis de ces 3 souches étant similaires à celui du traitement témoin quant à la capacité de formation de nodules, présagent de leur non efficience.

Les plus fortes amplitudes de production de nodules secs ayant été obtenues avec les variétés Doko et Boa-Vista inoculées par les souches 26D4, IRAT-FA3 et 81R1, ces variétés et ces souches sont dites compatibles. Ce qui pourrait expliquer comme chez le niébé, les quantités importantes de nodules produites par certaines variétés cultivées en Côte d'Ivoire (Konaté et al., 2013).

Au niveau de la hauteur d'insertion des premières gousses sur la tige principale, les travaux de Ziqiang et Danying (2002) ont montré que cette variable est étroitement liée à la taille du plant. Selon ces auteurs, plus une variété a des plants de grande taille, plus le niveau d'insertion des premières gousses sur la tige principale est important. Les résultats obtenus dans ces travaux confirment cette corrélation établie par ces auteurs. En effet, les variétés Doko, Boa-Vista et Tracaja ont été décrites par (N'Gbesso, 2011) comme étant des variétés de grandes tailles. Elles ont eu effectivement les meilleurs niveaux d'insertion des gousses. De même, N'Gbesso et al. (2013) ont montré que les variétés de soja telles R8-271; 1878-12E; R2-231et $1740-2 \mathrm{~F}$ qui ont une croissance importante ont affiché un bon niveau d'insertion des premières gousses pour être adaptées à la récolte mécanisée. Miles et Chen (2001) ont aussi montré dans des essais similaires conduits au Canada que les variétés de soja Shironomai et Kegon qui ont eu les plants de plus grandes tailles sur les 16 variétés étudiées ont eu aussi de bons niveaux d'insertion des gousses avec respectivement $14,2 \mathrm{~cm}$ et 13,5 $\mathrm{cm}$. Elles ont aussi affiché les meilleures biomasses par plant, les meilleurs poids de 100 gousses à une, deux et trois graines. ChinCheng et Chung-Sheng (2008) ont estimé entre 5 et $7 \%$ les pertes de récolte liées à une mauvaise sélection au niveau de ce caractère comme il aurait pu en être dans certains cas de maladies comme les pustules bactériennes (Zinsou et al., 2015). En outre, Shanmugasundaraman et Miao-Rong (2002) ont situé l'importance de ce caractère dans la 
réduction du temps de travail et du coût de production de $40 \%$ qu'elle procure avec la mécanisation de la récolte. Le soja ainsi produit devient très compétitif sur le marché.

Le niveau de couverture du sol se traduit par l'envergure des plants chez les légumineuses comme le soja. Il est strictement lié au développement végétatif de ces derniers grâce aux nodules qui sont de grandes pourvoyeuses d'azote qui provoque une croissance rapide des plants. Ce qui agit favorablement sur la production de biomasses et par conséquent, sur l'envergure et la capacité de couverture du sol par les variétés (Issoufou et al., 2017). Cependant, selon Karaboneye (2013) une bonne nodulation n'est pas toujours synonyme d'une bonne fixation d'azote car les nodules observés peuvent ne pas être tous efficients.

\section{Conclusion}

L'étude sur l'efficacité symbiotique de cinq souches locales de rhizobiums a permis d'obtenir dans l'ensemble des résultats probants au niveau de chaque paramètre de croissance étudié. Ainsi, des délais de floraison de certaines variétés ont été fortement influencés par certaines souches de bactéries. Pour d'autres, au contraire aucun effet n'a été constaté à ce niveau. Les délais de maturité étaient compris entre 96,33 jours pour les traitements précoces et 115,3 jours pour les plus tardifs. La plus grande différence de taille à maturité $(18,7 \mathrm{~cm})$ a été observée avec les traitements de la souche 26D4 au niveau de la variété Tracaja. En ce qui concerne le poids sec de nodules, toutes les variétés à l'exception de la variété Piramama, ont réagi positivement en produisant des quantités de nodules selon la souche de bactérie inoculée. Les poids obtenus ont varié de 4,3 g avec la variété Canarana inoculée par la souche IRAT-FA3 à 10,8 g avec la variété Boa-Vista inoculée par 26D4. La plupart des variétés n'ont pas répondu à l'inoculation des souches $11 \mathrm{C} 1,68 \mathrm{D} 2$ et $5 \mathrm{CB}$. Les meilleurs niveaux de couverture du sol et de longueur de tige ont été obtenus avec les traitements issus des souches 26D4 et 81R1. En fonction des résultats obtenus seules les ouches 26D4 et $81 \mathrm{R} 1$ ont montré une efficacité symbiotique sur la plupart des paramètres de croissance chez le soja. Ces deux souches vont être utilisées pour produire des inoculums au laboratoire.

\section{CONFLIT D'INTERETS}

Les auteurs de cet article déclarent sur l'honneur qu'il n'existe aucun conflit d'intérêts entre eux.

\section{CONTRIBUTIONS DES AUTEURS}

MFDPN a conduit les expérimentations et a préparé le manuscrit. LF a participé à la correction et la mise en forme du manuscrit; il est le chef du programme de recherche sur les plantes maraîchères et protéagineuses. NDC a effectué l'analyse statistique des données et la correction du manuscrit. NCK a participé à la correction et à la mise en forme du manuscrit; il est le collaborateur extérieur au programme de recherche sur les plantes maraîchères et protéagineuses.

\section{REMERCIEMENTS}

Que les Responsables du Centre National de Recherche Agronomique trouvent en cet article le couronnement d'un long processus d'activités dont ils ont autorisé la réalisation.

\section{REFERENCES}

Aboubacar K, Ousmane ZM, Amadou HI, Zouberou AM. 2013. Effet de la coinoculation $\mathrm{du}$ rhizobium et de mycorhizes sur les performances agronomiques du niébé [Vigna unguiculata (L.) Walp] au Niger. $J$. Appl. Biosci., 72 : 5846-5854. DOI : http://dx.doi.org/10.4314/jab.v72i1.9967 2

Anikwe MAN, Okonkwo CI, Mbah CN. 2003. Nodulation Effectivity, N-Accumulation 
and Yield of Soybean (Glycine max) in a Clayloam Soil Treated with Pre- and Post-Emergence Herbicides.

Tropicultura, 21: 22 - 27.

Avit JB, Pedia PL, Sankaré Y. 1999. Diversité Biologique de la Côte d'Ivoire. Rapport de synthèse, Ministère de l'Environnement et de la Forêt $273 \mathrm{p}$

AVRDC. 2009. Manual of vegetable soybean characterization and evaluation-Research Needs for Production and Quality Improvement, Taiwan $111 \mathrm{p}$

Brindou BY. 2016. Effet de trois substrats sur deux variétés de tomate en culture hors-sol au centre de la Côte d'Ivoire. Mémoire de Master 2, Université Nangui Abrogoua, Abidjan Côte d'Ivoire 52p

Brou YT, Akidess G, Bigot S. 2005. La variabilité climatique en Côte d'Ivoire : Entre perceptions sociales et réponses agricoles. Cah. Agric., 14(6): 533-540.

Chin-Chen H, Chung-Sheng S. 2008. Management inputs and mechanical harvesting of soybean in Taiwan. In Vegetable Soybean Research Needs for Production and Quality Improvement, Shanmugasundaram S (ed). AVRDC: Taiwan; 61-64.

Fondio L, Djidji AH, N'Gbesso MFP, Koné D. 2013. Evaluation de neuf variétés de tomate (Solanum Lycopersicum L.) par rapport au flétrissement bactérien et à la productivité dans le Sud de la Côte d'Ivoire. Int. J. Biol. Chem. Sci., 7(3): 1078-1086.

DOI http://dx.doi.org/10.4314/ijbcs.v7i3.15

Gbaguidi AA, Assogba P, Dansi M, Yedomonhan H, Dansi A. 2015. Caractérisation agromorphologique des variétés de niébé cultivées au Bénin. Int. J. Biol. Chem. Sci., 9(2): 1050-1066. DOI: http://dx.doi.org/10.4314/ijbcs.v9i2.40.

Issouffou $\mathrm{HO}$, Boubacar $\mathrm{S}$, Adam $\mathrm{T}$, Boubacar Y. 2017. Identification des insectes, parasites et évaluation économique de leurs pertes en graines sur les variétés améliorées et locale de niébé en milieu paysan à Karma. Int. J. Biol. Chem. Sci., 11(2): 694-706. DOI: https://dx.doi.org/10.4314/ijbcs.v11i2.13

Karaboneye F. 2013. Caractérisation de l'efficacité symbiotique des lignées africaines de soja à haute promiscuité. Mémoire de Maîtrise en Biologie. Université de Laval, Québec, Canada $123 \mathrm{p}$.

Konaté Z, Messoum FG, Sékou A, YaoKoumé A, Camara M, Keli Z J. 2013. Effets des cultures de soja (Glycine max) et de niébé (Vigna unguiculata) sur la densité apparente et la teneur en eau des sols et sur la productivité du riz pluvial de plateau sur ferralsol hyperdystrique : cas de Gagnoa, au Centre-Ouest de la Côte d'Ivoire. Int. J. Biol. Chem. Sci., 9(5): 2377-2384. DOI: http://dx.doi.org/10.4314/ijbcs.v7i1i.5

Koné B, Diatta S, Oikeh S, Gballou Y, Camara M, Dohm DD, Assa A. 2009. Estimation de la fertilité potentielle des ferralsols par la couleur: Usage de la couleur en morphopédologie. Canadian Journal of Soil Science, 89(3): 331-342. https://doi.org/10.4141/CJSS08042

Kouamé NC, Fondio L, Djidji AH, N'Gbesso MFP. 2002. Rapport d'activités de recherche pour le développement de la culture du soja dans les zones Centre et Centre-Nord de la Côte d'Ivoire. Convention CNRA-PROJET PACIL, $105 p$.

Kouamé NC, Fondio L, Djidji AH, N'Gbesso MFP. 1994. Rapport d'activités de recherche pour le développement de la culture du soja dans les zones Nord et Nord-Ouest de la Côte d'Ivoire. Convention CNRA-PROJET SOJA, 85p.

Lecourtier M. 2016. L'inoculation, clé du développement? Cultivar 18p. 
Miles CA, Chen C. 2001. Edamame variety trials in western Washington. WSU Vancouver

Research and Extension Unit, 1919 NE $78^{\text {th }}$ Street, Vancouvert WA 98665, USA, 4p.

Mulongoy K. 2005. Biological nitrogen fixation. Technical paper 2, South Dakota State University. Plant Science Department; 13 p.

N'Gbesso MFP, Kouamé NC, Zohouri GP, Konaté D. 2013. Evaluation finale du rendement et des paramètres phytosanitaires de lignées de soja [Glycine max (L.) Merrill] dans deux zones agro écologiques de savane de Côte d'Ivoire. Int. J. Biol. Chem. Sci., 7(2) : 574-583. DOI: http://dx.doi.org/10.4314/ijbcs.v7i2.14

N'Gbesso MFP. 2011. Thèse de doctorat unique $\mathrm{N}^{\circ}$ 682/2011. UFR Biosciences Université de Cocody-Abidjan, 187p.

N'Gbesso MFP, Kouamé NC, N'Guetta ASP, Foua BK. 2010. Evaluation [Glycine $\max (\mathrm{L}$.$) Merrill]. Sciences et$ Nature, 7(1): 59-67.

Soro S, Doumbia M, Dao D, Tschannen A, Giradin O. 2007. Performance de six cultivars de tomate Lycopersicon esculentum (Mill.) contre la jaunisse en cuillère des feuilles, le flétrissement bactérien et les nématodes à galles. Sciences et Nature, 4(2) : 123-130.

Ouattara N. 2001. Note Thématique sur les Ressources Génétiques Forestières. Situation des ressources génétiques forestières de la Côte d'Ivoire (Zone de savanes), $43 \mathrm{p}$.
Shanmugasundaram S, Miao-Rong Y. 2002. Mechanization of Vegetable Soybean Production in Taiwan. Asian Vegetable Research and Development Center (AVRDC), Shanhua, 741, Taiwan, Annual report, 59p.

Woomer PL. 2010. Fixation Biologique et Entreprise de légumineuses à graines : Directives des Maîtres Fermiers pour $\mathrm{N}_{2}$ Africa. Tropical Soil Biology and Fertility Institute of the International Centre for Tropical Agriculture, 17p.

World G. 2013. Côte d'Ivoire: divisions administratives (population et superficie). Disponible auprès de : http://www.gazetteer.de/wg.php? $\mathrm{x}=$ $\&$ men $=$ gadm $\& \operatorname{lng}=$ fr $\&$ des $=$ wg $\&$ geo $=50$ $\&$ srt=npan\&col=abcdefghinoq \&msz = 1500. Novembre 2016.

Zinsou VA, Zoumarou-Wallis N, Afouda ACL, Pate-Bata BT, Albert Kora SA, Dossou L, Aflokou F, Ahohuendo B. 2015. Effet des dates de semis sur les niveaux de sévérité de la pustule bactérienne (Xanthomonas axonopodis pv. glycines) de soja (Glycine max) au Nord Bénin. Int. J. Biol. Chem. Sci., 9(5): 2377-2384. DOI: http://dx.doi.org/10.4314/ijbcs.v9i5.10

Ziqiang W, Danying W. 2002. Studies on the correlation between the quality traits of vegetable soybean. Annual report. Dept. of Agronomy. Zhejiang University, China, 5 p. 